\title{
Optimization of cable-stayed bridges with box-girder decks
}

\author{
L.M.C. Simões* , J.H.J.O. Negrão \\ Department of Civil Engineering, University of Coimbra, 3049 Coimbra Codex, Portugal
}

\begin{abstract}
Box-girder decks are very effective solutions for long span cable-stayed bridges, due to their high torsional stiffness and streamlined profile, which usually lead to a good aerodynamic behaviour. A study on the optimization of such structural system is presented in this paper. The deck is modelled through the assembly of planes of plate-membrane elements. A multicriteria approach is considered for the optimization itself, with constraints on maximum stresses, minimum stresses in stays and deflections under dead load condition. Two illustrative examples are shown. (C) 2000 Elsevier Science Ltd. All rights reserved.
\end{abstract}

Keywords: Cable-stayed bridges; Optimization; Box-girder decks

\section{Introduction}

Cable-stayed bridges are large and sophisticated structures which may greatly benefit from the use of structural optimization techniques for preliminary design improvement. General optimization packages are not appropriate for this purpose given the special characteristics of this structural system and the rigorous aesthetical and serviceability design. Therefore, an integrated analysis-optimization application for this type of structure was developed by the authors, in which the required adaptations were implemented at the code level. The programme was named cablestayed bridges integrated analysis and optimization (CIAO).

This programme was tested in the investigation of some relevant aspects that affect the design of a cable-stayed bridge. The research model on shape and sizing optimization of cable-stayed bridges started by using a 2D finite element model for the analysis [1]. The problem was extended to three-dimensional analysis and consideration of erection stages under static loadings [2,3]. Seismic effects must be considered in structural design in earthquake-prone countries. Therefore, a reliable solution requires the consideration of this loading at the early stage of optimization. A sensitivity analysis algorithm derived from the modal-spectral approach in connection with the complete quadratic combination method (CQC) [4] was later described [5]. However, in instances such as with strongly non-linear behaviour or when coalescence situations arise, the algorithm may not be valid or lead to runtime error. The alternative use of a time-history based sensitivity analysis

\footnotetext{
* Corresponding author.
}

procedure is then recommended, though computationally expensive. Such algorithm was also implemented in the software. Recent papers [6,7] discuss the specific issues and relative merits of both methods.

\section{Analysis model}

\subsection{Deck}

In most of the previous studies, a grid solution was adopted for modelling the deck, with side stiffening girders supporting transverse beams. However, box-girder decks provide increased torsional resistance and better aerodynamic behaviour than open sharp deck profiles, which are decisive aspects in the design of long-span cable-stayed bridges. Besides, when certain structural solutions such as the single plan arrangement of cables are adopted, the use of box-girder sections is more appropriate.

This paper concerns the optimization of cable-stayed bridges with this type of deck. Prior to the code implementation, one had to choose the numerical model to be used from among three possible types: (i) "spine" discretization, in which the deck is modelled by a fictitious beam with global stiffness characteristics similar to those of the true box-girder cross section; (ii) specific box-girder element formulation; (iii) assembly of plate-membrane plane elements.

The former approach was used in an early paper of the authors [1], although the two-dimensional analysis undertaken in that study did not allow for the consideration of out-of-plane behaviour. However, in spite of being computationally affordable, this model requires that 


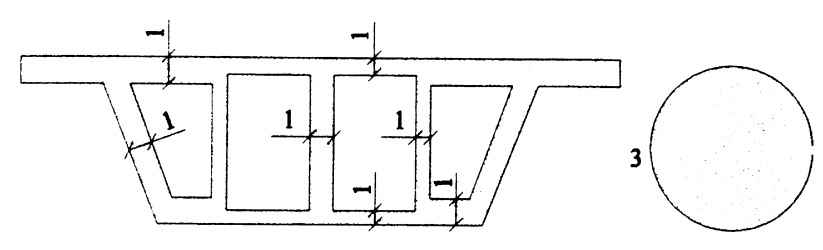

Fig. 1. Sizing design variables.

pre- and post-processing is provided to set the fictitious beam dimensions and to account for box-girder effects such as warping and shear-lag. Furthermore, as far as optimization is concerned, a two-level procedure must be used. Fictitious cross-sectional areas and inertias are used as intermediate design variables from which the optimal values of the primary ones - plate thicknesses and cell dimensionsare in turn optimized.

Specific box-girder elements intend to conciliate computational effectiveness and analysis accuracy. Nukulchai and Hong [8] formulate one such class of elements. However, a different number of degrees of freedom is assigned to the element nodes, which may require some major changes of the analysis code. Besides, these elements are usually not available in the libraries of most f.e. packages and therefore its full development is required. Finally, the elements lack flexibility for modelling different cross-section geometries, such as multicell box-girders, side cantilever plates and diaphragms.

The latter approach was, therefore, selected for use in the programme. Given its suitability for both thin or thick plate problems, Reissner-Mindlin formulation was considered and 4- and 9-noded isoparametric lagrangean platemembrane elements were developed. A selective quadrature procedure was used for the stiffness matrix, with a $2 \times 2$ rule for the bending terms and a single point rule for shear and membrane sub-matrices. A fictitious in-plane rotational stiffness was added to coplanar nodes in order to prevent system singularity. Null-energy modes may arise from the use of selective quadrature, causing the solution process to fail. However, in the specific type of structure under study, they were found not to propagate through the mesh, due to the intersection of box-girder element planes and the boundary conditions at the extreme cross-sections.

\subsection{Stays}

Most cable-stayed bridges show moderate non-linear behaviour, which is contributed, from three main sources: the sag effect of the cables, the effect of large displacements and $\mathrm{P}-\Delta$ interaction. Therefore, some provisions to account for non-linear effects need to be considered and the use of a geometrically non-linear analysis approach should look the obvious choice. However, although the two latter effects may play an important role in instances such as alternate loading in large spans and/or erection stages, most authors agree in that the catenary effect of the cables is the dominant source of non-linearity. Besides, one is to expect very high computational costs and considerable numerical difficulties in implementing sensitivity analysis for a pure geometrically non-linear approach.

Therefore, the equivalent or Ernst modulus method, which accounts for the non-linear behaviour of the stays while still allowing for the use of a pseudo-linear analysis, was chosen. The main problem concerning this method is that different equivalent modulus must be assigned to each cable for each load case (according to its actual stress condition) when several load conditions are considered. This would require the equation solver to be restarted for each load case, which is extremely expensive in a single analysis process and even more in an optimization context. Fortunately, the variation of the equivalent modulus within the range of cable stresses spanning the various load cases is usually small. In fact, large minimum tensile stresses are required to act in the stays, in order to provide them with the adequate stiffness. Such type of condition can be added as a constraint in the optimization formulation, to guarantee a minimum level of stress.

Thus, an averaged equivalent instantaneous Ernst modulus was used, for each stay, for all the load cases under consideration. When erection stages are accounted for, the stress amplitude from one stage to another may be considerably large and the mean stress is usually smaller than average stress acting in the cable in the final structure. In such case, since the previous approach is no longer valid, a secant equivalent Ernst modulus whose value is derived from the stress in both the current and the following stage is used instead. However, this topic shall not be discussed in detail in this paper.

\section{Design variables}

Selecting a trial design for a cable-stayed bridge involves setting a large number of parameters of both sizing, shape and mechanical (prestressing) types. Owing to the complex

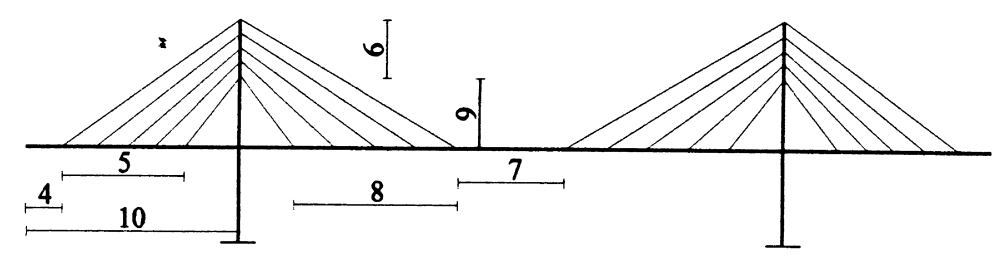

Fig. 2. Shape design variables. 


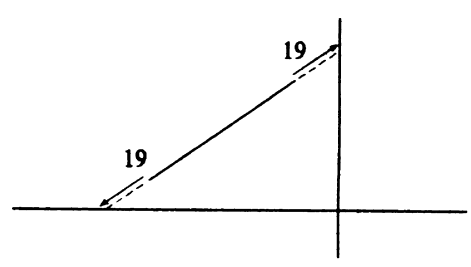

Fig. 3. Fixed-end prestressing design variable.

structural behaviour and parameter interaction, it is not easy to foresee how to combine their values in order to achieve a reasonably effective and feasible design. For the optimization sake, it is desirable that a wide choice of potential design variables is available. This was achieved by introducing the concept of design variable library. Basically, it consists of a set of procedures and instructions on how to compute goals and sensitivities for a set of previously defined parameters. These concern geometrical description for a number of cross-section shapes, overall structural geometry and cable prestresses. Figs. 1-3 show the relevant design variable types for the kind of problem being discussed. Other types are available for plate girder type decks.

Different thicknesses may be assigned to the various panels of the box-girder cross section. Shape cross-sectional design variables such as box height or bottom width, which may play an important role in future research concerning seismic or aerodynamics problems, are currently under development.

Maximum structural cost decrease is achieved with formulations in which the three types of design variables are combined. Sizing design variables provide for direct volume/cost reduction. Shape and prestressing design variables, with respect to which cost is almost insensitive, play nevertheless an important role in allowing for better stress distribution, leading to additional sizing reductions. Prestressing design variables may be considered alone for the problem of optimal correction of cable forces during erection [9].

Several trial designs may be tested by simply switching on or off each type of design variable or its assignment to specific subdomains or groups of elements. This intensive data process is automatically handled by the programme. Pre- and post-processing routines are available for initial generation and updating of the f.e. mesh and variable linking when shape design variables are considered. However, care must be taken to avoid excessively narrow plate elements in the transverse direction, which causes degeneration of the numerical accuracy. This may happen because longitudinal discretization accounts for both the cable anchorage positions, which vary throughout the process, and the diaphragms spacing, which is likely to be a prescribed value. In such cases, a refined discretization must be used for both directions, which results in the increase of the computational cost.

\section{Sensitivity analysis}

\subsection{General}

Given the availability of the source code, the discrete nature of cable-stayed bridge structures and the large number of constraints (stresses and displacements) under control, the analytical discrete direct method was used for the sake of sensitivity analysis. The method relies upon the differentiation of the finite element equilibrium equation set

$\mathbf{K} \frac{\mathrm{d} \mathbf{u}}{\mathrm{d} x_{i}}=-\frac{\mathrm{d} \mathbf{P}}{\mathrm{d} x_{i}}-\frac{\mathrm{d} \mathbf{K}}{\mathrm{d} x_{i}} \mathbf{u}=\mathbf{Q}_{\mathbf{p} i}$

in which $\mathbf{Q}_{\mathrm{P}}$ is the virtual pseudo-loading corresponding to design variable $x_{i}$.

\subsection{Plate-membrane element sensitivity analysis}

The coefficients of the f.e. equation system result from assembling the element contributions. Therefore, the same applies to their derivatives in Eq. (1), which requires sensitivity analysis to be developed at the element level.

Plate-membrane elements of the deck show explicit dependency only on design variables types $1,4,5,7,8$, 10 of Figs. 1 and 2. Type 1 is a sizing design variable and the remaining types are shape design variables. Sensitivitity analysis is basically different for each of these groups and a brief description shall be made in the following.

From the f.e. formulation of Reissner-Mindlin platemembrane elements, one can notice that the coefficients of the element stiffness matrix are of either of the forms

$\int_{\Omega} F(h) N_{i}(x, y) N_{j}(x, y) \mathrm{d} x \mathrm{~d} y$

$\int_{\Omega} F(h) \frac{\mathrm{d} N_{i}(x, y)}{\mathrm{d} s} N_{j}(x, y) \mathrm{d} x \mathrm{~d} y \quad \mathrm{~s}=x$ or $y$

$\int_{\Omega} F(h) \frac{\mathrm{d} N_{i}(x, y)}{\mathrm{d} s} \frac{\mathrm{d} N_{j}(x, y)}{\mathrm{d} r} \mathrm{~d} x \mathrm{~d} y \quad \mathrm{~s}=x$ or $y, \mathrm{r}=x$ or $y$

in which $h$ is the plate thickness, $(x, y)$ are the element node cartesian local co-ordinates and $N_{j}$ are the element shape functions.

For the purpose of systematic application, Eqs. (2a)-(2c) are usually expressed in terms of intrinsic co-ordinates, leading to

$$
\begin{aligned}
& \int_{-1}^{1} \int_{-1}^{1} F(h) N_{i}(\xi, \eta) N_{j}(\xi, \eta)|\mathbf{J}| \mathrm{d} \xi \mathrm{d} \eta \\
& \int_{-1}^{1} \int_{-1}^{1} F(h) f^{\mathrm{s}}\left(\xi, \eta, \mathbf{x}_{\mathrm{e}}\right) N_{j}(\xi, \eta) \mathrm{d} \xi \mathrm{d} \eta \quad \mathrm{s}=x \text { or } y
\end{aligned}
$$


$\int_{-1}^{1} \int_{-1}^{1} F(h) f^{\mathrm{s}}\left(\xi, \eta, \mathbf{x}_{\mathrm{e}}\right) f^{\mathrm{r}}\left(\xi, \eta, \mathbf{x}_{\mathrm{e}}\right) \frac{1}{|\mathbf{J}|} \mathrm{d} \xi \mathrm{d} \eta$

$\mathrm{s}=x$ or $y \mathrm{r}=x$ or $y$

where $\mathbf{J}$ is the Jacobian matrix and

$f_{k}^{x}\left(\xi, \eta, \mathbf{x}_{\mathrm{e}}\right)=\left(\frac{\partial y}{\partial \eta} \frac{\partial N_{k}}{\partial \xi}-\frac{\partial y}{\partial \xi} \frac{\partial N_{k}}{\partial \eta}\right)$

for differentiation with respect to $x$

$f_{k}^{y}\left(\xi, \eta, \mathbf{x}_{\mathrm{e}}\right)=\left(-\frac{\partial x}{\partial \eta} \frac{\partial N_{k}}{\partial \xi}+\frac{\partial x}{\partial \xi} \frac{\partial N_{k}}{\partial \eta}\right)$

for differentiation with respect to $y$

The derivatives of the cartesian local co-ordinates are derived from the isoparametric relations involving the element $N$ nodes co-ordinates

$x=\sum_{j=1}^{N} N_{j}(\xi, \eta) x_{j} \quad y=\sum_{j=1}^{N} N_{j}(\xi, \eta) y_{j}$

$\frac{\partial x}{\partial \xi}=\sum_{j=1}^{N} \frac{\mathrm{d} N_{j}}{\mathrm{~d} \xi} x_{j} \frac{\partial y}{\partial \xi}=\sum_{j=1}^{N} \frac{\mathrm{d} N_{j}}{\mathrm{~d} \xi} y_{j}$

$\frac{\partial x}{\partial \eta}=\sum_{j=1}^{N} \frac{\mathrm{d} N_{j}}{\mathrm{~d} \eta} x_{j} \frac{\partial y}{\partial \eta}=\sum_{j=1}^{N} \frac{\mathrm{d} N_{j}}{\mathrm{~d} \eta} y_{j}$

By differentiation of Eqs. (3a)-(3c) with respect to the design variable $x_{i}$ one can get now the sensitivities of the stiffness coefficients.

For the case in which a sizing design variable (plate thickness) is being considered, only the function $F(h)$ which sets strain variation through the plate depth leads non-zero derivative and the sensitivity shall be readily available.

When dealing with shape design variables, all the remaining terms in Eqs. (3a)-(3c) are variable dependant and the sensitivity expression results somewhat more complex:

$$
\begin{aligned}
& \int_{-1}^{1} \int_{-1}^{1} F(h) N_{i}(\xi, \eta) N_{j}(\xi, \eta) \frac{\mathrm{d}|\mathbf{J}|}{\mathrm{d} x_{i}} \mathrm{~d} \xi \mathrm{d} \eta \\
& \int_{-1}^{1} \int_{-1}^{1} F(h) \frac{\mathrm{d} f^{\mathrm{s}}\left(\xi, \eta, \mathbf{x}_{\mathrm{e}}\right)}{\mathrm{d} x_{i}} N_{j}(\xi, \eta) \mathrm{d} \xi \mathrm{d} \eta \\
& \int_{-1}^{1} \int_{-1}^{1} F(h)\left[\frac{\mathrm{d} f^{\mathrm{s}}\left(\xi, \eta, \mathbf{x}_{\mathrm{e}}\right)}{\mathrm{d} x_{i}} f^{\mathrm{r}}\left(\xi, \eta, \mathbf{x}_{\mathrm{e}}\right)\right. \\
& +f^{\mathrm{s}}\left(\xi, \eta, \mathbf{x}_{\mathrm{e}}\right) \frac{\mathrm{d} f^{\mathrm{r}}\left(\xi, \eta, \mathbf{x}_{\mathrm{e}}\right)}{\mathrm{d} x_{i}} \\
& \left.-f^{\mathrm{r}}\left(\xi, \eta, \mathbf{x}_{\mathrm{e}}\right) f^{\mathrm{s}}\left(\xi, \eta, \mathbf{x}_{\mathrm{e}}\right) \frac{1}{|\mathbf{J}|} \frac{\mathrm{d}|\mathbf{J}|}{\mathrm{d} x_{i}}\right] \frac{1}{|\mathbf{J}|} \mathrm{d} \xi \mathrm{d} \eta
\end{aligned}
$$

One has for the sensitivity of the Jacobian determinant

$$
\begin{aligned}
\frac{\mathrm{d}|\mathbf{J}|}{\mathrm{d} x_{i}}= & \frac{\mathrm{d}\left(\frac{\partial x}{\partial \xi}\right)}{\mathrm{d} x_{i}} \frac{\partial y}{\partial \eta}+\frac{\partial x}{\partial \xi} \frac{\mathrm{d}\left(\frac{\partial y}{\partial \eta}\right)}{\mathrm{d} x_{i}}-\frac{\mathrm{d}\left(\frac{\partial x}{\partial \eta}\right)}{\mathrm{d} x_{i}} \frac{\partial y}{\partial \xi} \\
& -\frac{\partial x}{\partial \eta} \frac{\mathrm{d}\left(\frac{\partial y}{\partial \xi}\right)}{\mathrm{d} x_{i}}
\end{aligned}
$$

By differentiation expressions for functions $f$ in Eqs. (4a) and (4b):

$$
\begin{aligned}
\frac{\mathrm{d} f_{k}^{x}}{\mathrm{~d} x_{i}} & =\frac{\mathrm{d}\left(\frac{\partial y}{\partial \eta}\right)}{\mathrm{d} x_{i}} \frac{\partial N_{k}}{\partial \xi}-\frac{\mathrm{d}\left(\frac{\partial y}{\partial \xi}\right)}{\mathrm{d} x_{i}} \frac{\partial N_{k}}{\partial \eta} \\
\frac{\mathrm{d} f_{k}^{y}}{\mathrm{~d} x_{i}} & =-\frac{\mathrm{d}\left(\frac{\partial x}{\partial \eta}\right)}{\mathrm{d} x_{i}} \frac{\partial N_{k}}{\partial \xi}+\frac{\mathrm{d}\left(\frac{\partial x}{\partial \xi}\right)}{\mathrm{d} x_{i}} \frac{\partial N_{k}}{\partial \eta}
\end{aligned}
$$

Derivatives of cartesian local co-ordinates in Eqs. (8) and (9) are finally obtained from differentiation of Eq. (6), resulting in:

$$
\begin{array}{ll}
\frac{\mathrm{d}}{\mathrm{d} x_{i}}\left(\frac{\partial x}{\partial \xi}\right)=\sum_{j=1}^{N} \frac{\mathrm{d} N_{j}}{\mathrm{~d} \xi} \frac{\mathrm{d} x_{j}}{\mathrm{~d} x_{i}} & \frac{\mathrm{d}}{\mathrm{d} x_{i}}\left(\frac{\partial y}{\partial \xi}\right)=\sum_{j=1}^{N} \frac{\mathrm{d} N_{j}}{\mathrm{~d} \xi} \frac{\mathrm{d} y_{j}}{\mathrm{~d} x_{i}} \\
\frac{\mathrm{d}}{\mathrm{d} x_{i}}\left(\frac{\partial y}{\partial \eta}\right)=\sum_{j=1}^{N} \frac{\mathrm{d} N_{j}}{\mathrm{~d} \eta} \frac{\mathrm{d} y_{j}}{\mathrm{~d} x_{i}} & \frac{\mathrm{d}}{\mathrm{d} x_{i}}\left(\frac{\partial x}{\partial \eta}\right)=\sum_{j=1}^{N} \frac{\mathrm{d} N_{j}}{\mathrm{~d} \eta} \frac{\mathrm{d} x_{j}}{\mathrm{~d} x_{i}}
\end{array}
$$

The derivatives of the nodal co-ordinates with respect to the shape design variables are, therefore, the basic brick to build all the required sensitivities. The particular shape of the domain, with all box-girder plate elements bounded by sides which are either parallel or orthogonal to the longitudinal direction makes that an easy task.

\section{Optimization}

\subsection{The algorithm}

NLP algorithms may face considerable difficulty in dealing with problems in which hundreds or thousands of constraints are to be controlled.

Since the simultaneous reduction of all the objectives (stresses and displacements) is desirable in structural optimization, one may employ a minimax approach, but these problems are discontinuous and non-differentiable and therefore difficult to solve. However, it may be shown $[10,11]$ that a minimax solution can be found by minimizing the unconstrained scalar function

$F(\mathbf{x})=\frac{1}{\rho} \ln \sum_{i=1}^{M} \mathrm{e}^{\rho g_{i}(\mathbf{x})}$ 


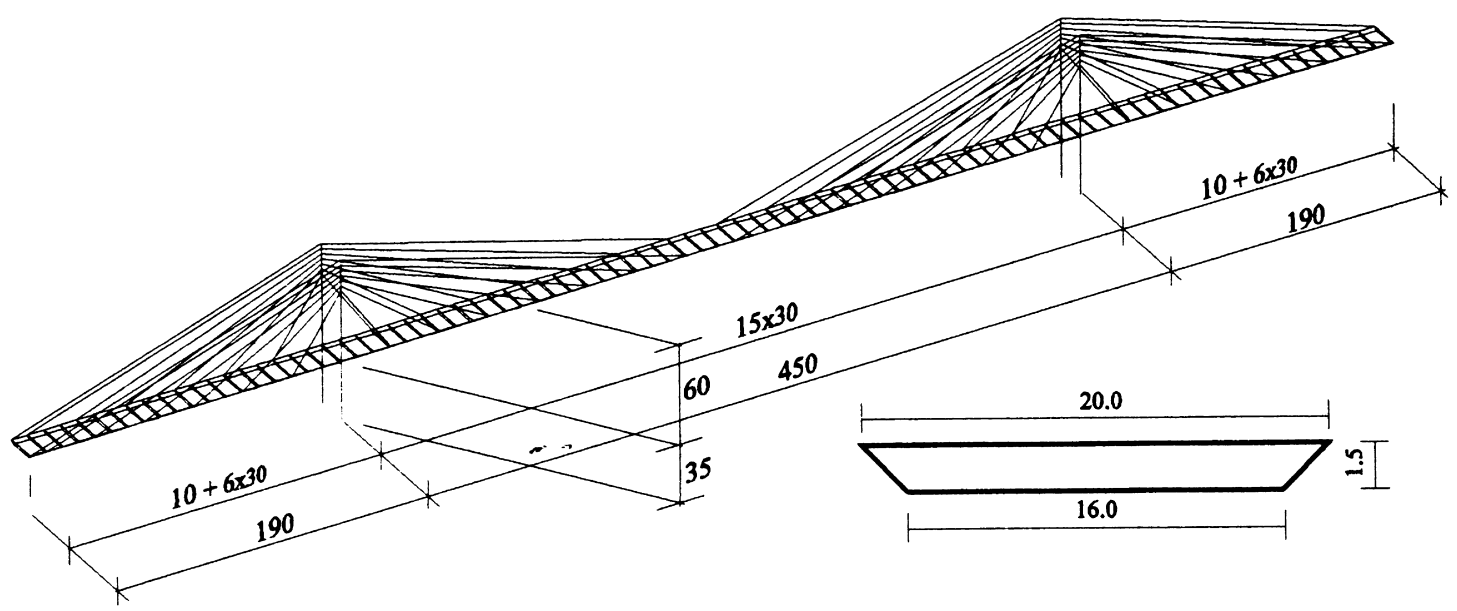

Fig. 4. Geometry of starting design.

or, in the explicit approximate form

$$
\begin{aligned}
F(\mathbf{x}) & =\frac{1}{\rho} \ln \sum_{i=1}^{M} \mathrm{e}^{\rho\left(g_{i}\left(\mathbf{x}_{0}\right)+\sum_{j=1}^{N} \frac{\partial g_{i}\left(\mathbf{x}_{0}\right)}{\partial x_{j}} \Delta x_{j}\right)} \\
& =\frac{1}{\rho} \ln \sum_{i=1}^{M} \mathrm{e}^{\rho\left(g_{i}\left(\mathbf{x}_{0}\right)+\nabla^{\mathrm{T}}\left(g_{i}\right) \Delta \mathbf{x}\right)}
\end{aligned}
$$

which is more suitable for numerical calculation. $N$ is the number of design variables, $g_{i}(\mathbf{x})$ are the $M$ objectives, $\nabla g(\mathbf{x})$ stands for objective gradient and $\Delta \mathbf{x}$ is the perturbation vector. $\rho$ is a user-defined parameter control which must be gradually increased throughout the iterative sequence.

\subsection{Constraints}

In order to prevent numerical inaccuracy, the objectives are turned into non-dimensional normalized form by using reference values which are:

- the volume/cost of the starting trial design;

- the allowable stress for materials;

- the tolerance deflection for geometry control.

According to this, the following types of objectives are formulated:

$g_{0}(\mathbf{x})=V / V_{0}-1 \leq 0 \quad$ Cost reduction goal

$g_{i}(\mathbf{x})=\sigma_{i} / \sigma_{A}-1 \leq 0 \quad$ Maximum allowable stress

$g_{i}(\mathbf{x})=\alpha-\sigma_{i} / \sigma_{A} \leq 0, \quad 0 \leq \alpha \leq 1$

Minimum required tensile stress in stays for effective stiffness

$g_{j}(\mathbf{x})=\delta_{j} / \delta_{0}-1 \leq 0 \quad$ Controlled deflections.

\section{Examples}

The process is illustrated by two examples that differ in the consideration of geometry control constraints (16).

The geometry of the starting design is shown in Fig. 4. A relatively large span had to be used in order to achieve significant stresses on deck, since the lower bound of $15 \mathrm{~mm}$, imposed for the equivalent thickness of the steel plates, result in a considerably large cross-sectional area. A single cell box-girder with the size shown is used. Diaphragms are $10 \mathrm{~m}$ spaced, in order to match all cable anchorage positions in both side and central spans. Minimum stress in stays is prescribed as $20 \%$ of allowable stress. For controlling the geometry in the dead load condition of problem 2, a tolerance of $5 \mathrm{~cm}$ is allowed for deflections at the deck anchorage points. Besides dead load condition needed for geometry control, another four load cases are considered: live load on side and/or central spans (3) and lateral wind (1). Dead load is taken as $2.0 \mathrm{kN} / \mathrm{m}^{2}$, additionally to structural self-weight. For live load a uniform load of $4.0 \mathrm{kN} / \mathrm{m}^{2}$ is considered, while as for wind a drag pressure of $1.5 \mathrm{kN} / \mathrm{m}^{2}$ is used, no lifting forces being accounted for.

Allowable stress is taken as $200 \mathrm{MPa}$ for pylons, $140 \mathrm{MPa}$ for deck plates and $500 \mathrm{MPa}$ for stays. 47 design variables were considered in both problems, with the following meaning and sequence:

1, 2, 3-equivalent thicknesses ( $\mathrm{mm}$ ) of top, bottom and side plates, respectively, of each side span outer $160 \mathrm{~m}$;

$4,5,6$ - the same, for $30 \mathrm{~m}$ on each side of the pylons;

$7,8,9$ - the same, for remaining part of central span;

10-13-length, width and thicknesses of pylons below the deck $(\mathrm{m}, \mathrm{m}, \mathrm{mm})$

14-17 - the same, for pylons above deck level

18-31-cross-sectional areas of cables, starting with the back-stays $\left(\mathrm{cm}^{2}\right)$

32-45-corresponding fixed-end prestressing forces in the cables $(\mathrm{MN})$

46 - length of end block zone of cables in pylons (m); 


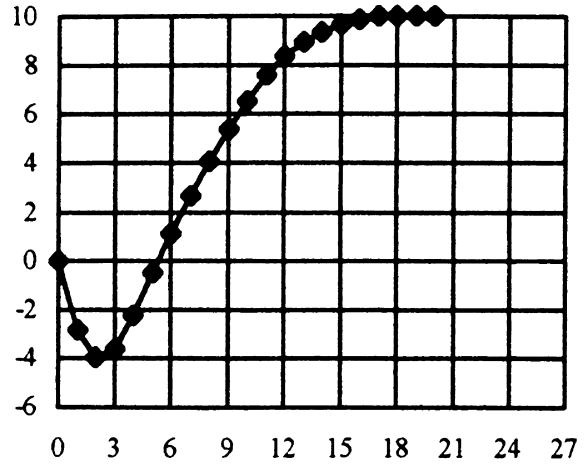

(a)

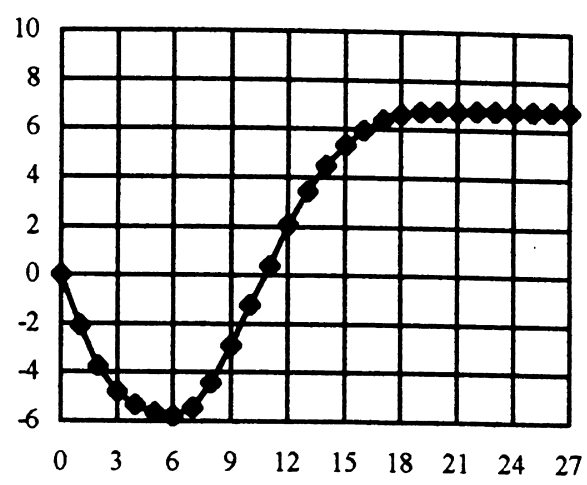

(b)

Fig. 5. Cost reduction (\%) against number of iterations: (a) no deflection control; and (b) with deflection control.
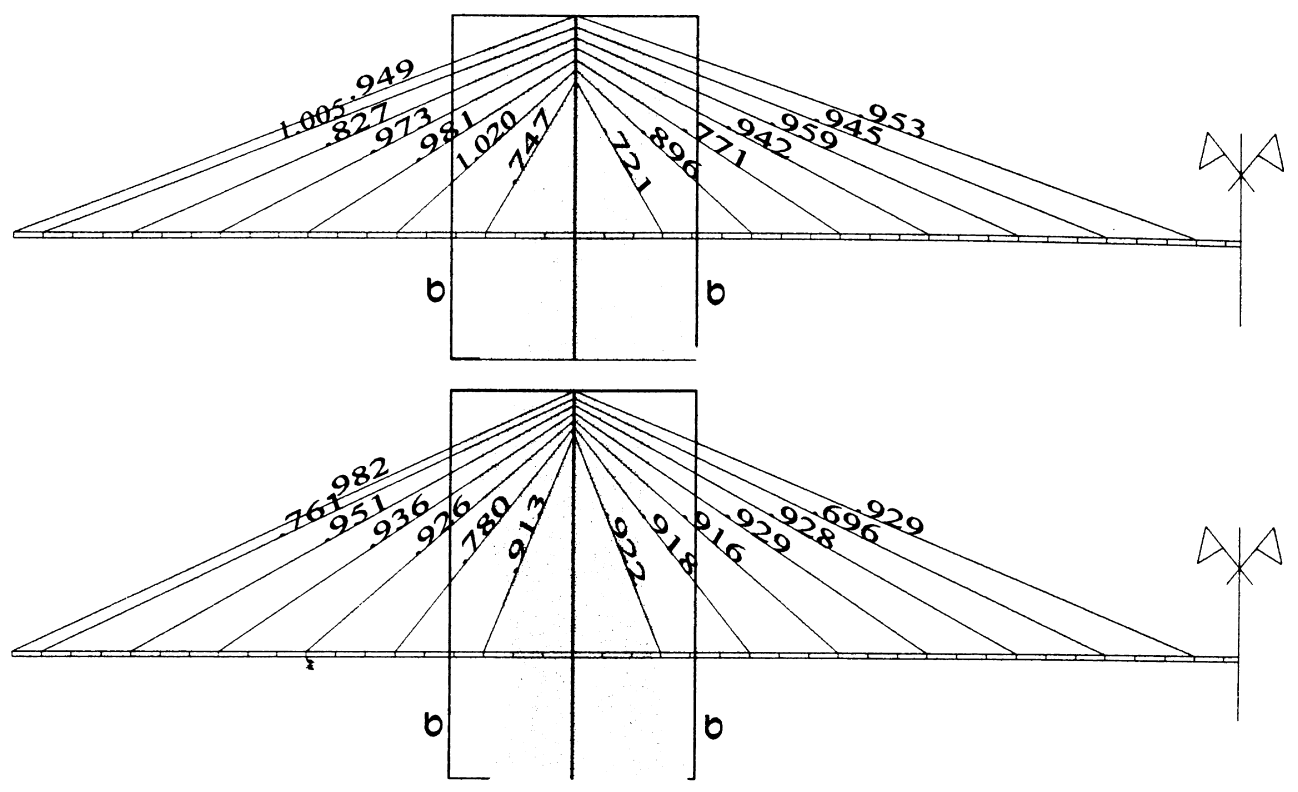

Fig. 6. Initial and final maximum stresses in stays and pylons.
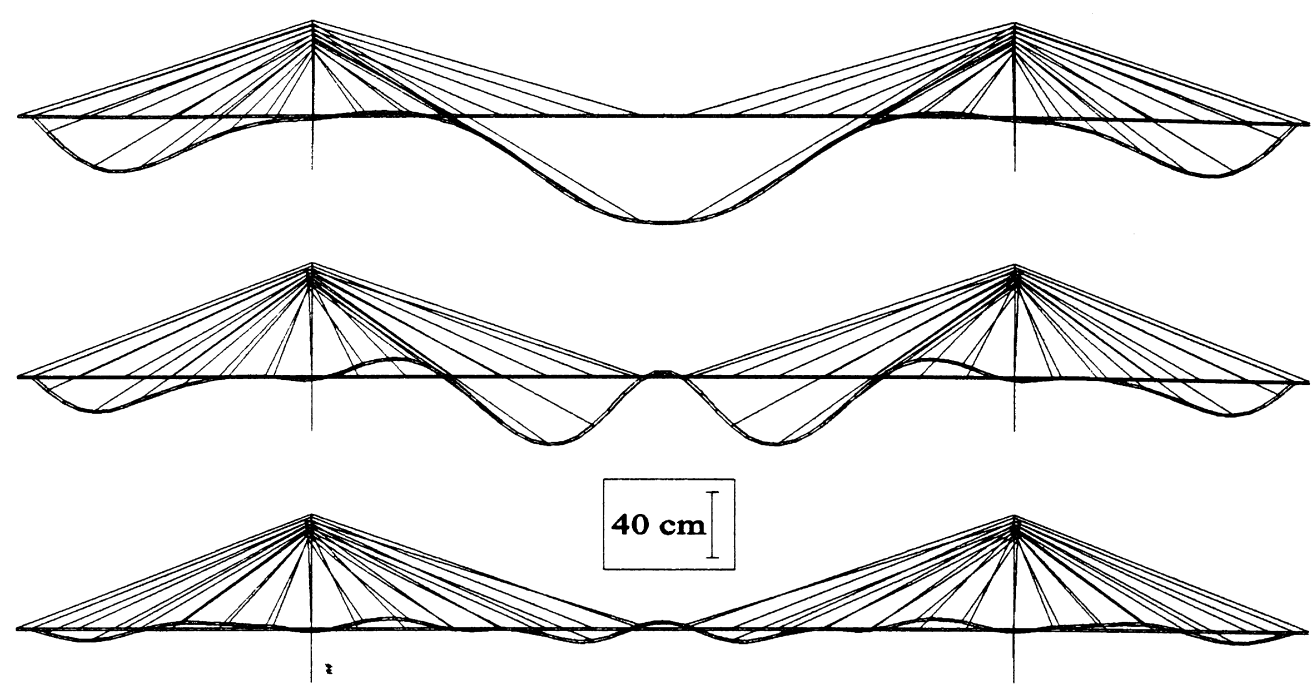

Fig. 7. Deflected shape for dead load condition in starting design and solutions 1 (no geometry control) and 2 (with geometry control). 
Table 1

Starting and final values of design variables for examples 1 and 2

\begin{tabular}{|c|c|c|c|}
\hline DV & St. & Ex1 & Ex2 \\
\hline 1 & 20.0 & 15.0 & 15.0 \\
\hline 2 & 20.0 & 15.0 & 15.0 \\
\hline 3 & 20.0 & 15.0 & 17.1 \\
\hline 4 & 20.0 & 15.0 & 15.0 \\
\hline 5 & 20.0 & 15.0 & 15.6 \\
\hline 6 & 20.0 & 15.0 & 15.0 \\
\hline 7 & 20.0 & 21.2 & 25.4 \\
\hline 8 & 20.0 & 16.3 & 15.0 \\
\hline 9 & 20.0 & 40.0 & 40.0 \\
\hline 10 & 3.50 & 5.00 & 5.00 \\
\hline 11 & 3.50 & 5.00 & 5.00 \\
\hline 12 & 40.0 & 15.0 & 15.0 \\
\hline 13 & 40.0 & 29.9 & 30.2 \\
\hline 14 & 3.50 & 5.00 & 5.00 \\
\hline 15 & 3.50 & 4.86 & 4.94 \\
\hline 16 & 40.0 & 15.0 & 15.0 \\
\hline 17 & 40.0 & 24.0 & 24.6 \\
\hline 18 & 350 & 390 & 326 \\
\hline 19 & 250 & 231 & 339 \\
\hline 20 & 150 & 75 & 78 \\
\hline 21 & 150 & 151 & 140 \\
\hline 22 & 150 & 108 & 121 \\
\hline 23 & 100 & 81 & 83 \\
\hline 24 & 100 & 68 & 72 \\
\hline 25 & 100 & 60 & 67 \\
\hline 26 & 100 & 106 & 129 \\
\hline 27 & 175 & 136 & 33 \\
\hline 28 & 175 & 126 & 208 \\
\hline 29 & 175 & 141 & 201 \\
\hline 30 & 200 & 45 & 44 \\
\hline 31 & 200 & 344 & 288 \\
\hline 32 & 10.0 & 15.0 & 12.6 \\
\hline 33 & 8.0 & 7.0 & 10.8 \\
\hline 34 & 3.0 & 2.3 & 3.1 \\
\hline 35 & 3.0 & 4.7 & 5.8 \\
\hline 36 & 3.0 & 4.0 & 5.7 \\
\hline 37 & 3.0 & 3.6 & 3.6 \\
\hline 38 & 3.0 & 3.2 & 3.8 \\
\hline 39 & 3.0 & 3.2 & 3.1 \\
\hline 40 & 3.0 & 4.7 & 4.4 \\
\hline 41 & 3.0 & 2.8 & 0.7 \\
\hline 42 & 3.0 & 0.0 & 5.3 \\
\hline 43 & 2.0 & 0.0 & 4.4 \\
\hline 44 & 2.0 & 0.0 & 0.4 \\
\hline 45 & 3.0 & 9.8 & 8.4 \\
\hline 46 & 18.0 & 12.0 & 12.0 \\
\hline 47 & 42.0 & 60.0 & 60.0 \\
\hline
\end{tabular}

47 - distance from deck level to the lowest cable end block (m).

Fig. 5 represents the cost reduction for both solutions. The starting design shows a constraint violation with respect to the minimum stress condition in the back-stays, when the live load on the side spans is acting on the structure. The optimization process looks for the design feasibility in the first place and that results in an initial increase in cost, which is recovered later. When deflection control is considered, the cost recovery takes place after a larger number of iterations. This is due to the arisal of several other constraint violations related to the deflection of controlled nodes, additionally to the minimum stress condition in the back-stays. As a result, the final cost reduction is just about $6.5 \%$ of the initial cost, against $10 \%$ for the example without deflection control.

Fig. 6 shows the initial and final stress distribution in the pylons and stays for example 1, similar results being achieved for example 2. However, the kinematic behaviour is rather different in each optimized design, as one can observe in Fig. 7. The deflected shape of problem 2 could still be enhanced if a smaller tolerance were prescribed for checking the controlled displacements. The sag of spans between consecutive stays depends only on local flexural behaviour and must be prevented by properly cambering the deck segments in the fabrication site (Table 1).

In both optimal designs the final arrangement of stays approaches the fan configuration, the end-block zone length converging to the lower bound.

Conversely, the distance from the deck to the lowest cable anchorage reaches the upper bound, which provides the stays with maximum slope and vertical stiffness.

\section{References}

[1] Simões LMC, Negrão JH. Sizing and geometry optimization of cablestayed bridges. Computers and Structures 1994;52:309-21.

[2] Negrão JH, Simões LMC. Optimization of cable-stayed bridges with three-dimensional modelling. Computers and Structures 1997;64:741-58.

[3] Negrão JH, Simões, LMC. Three dimensional nonlinear optimization of cable-stayed bridges. In: Topping B, Papadrakakis M, editors. CST/94 - advances in structural optimization. Proceedings of the Second International Conference on Computational Structures Technology, Athens, Greece, Civil-Comp, Edinburgh, 1994. p. 203-13.

[4] Wilson EL, Kiureghian A, Bayo EP. A replacement for the SRSS method in seismic analysis. Earthquake Engineering and Structural Dynamics 1981;9:187-92.

[5] Simões LMC, Negrão JH. Optimization of cable-stayed bridges subjected to dynamic loading. In: Olhoff N, Rozvany G, editors. WCSMO1. Proceedings of the First World Congress of Structural and Multidisciplinary Optimization, Goslar, Germany, Pergamon, Oxford, 1995. p. 797-802.

[6] Negrão JH, Simões LMC. Effectiveness of modal/spectral versus timehistory methods. In: IUTAM, ed. Optimization of cable-stayed bridges under seismic action, in 19th ICTAM. 19th International Congress on Theoretical and Applied Mechanics, Kyoto, Japan, 1996. p.268.

[7] Simões LMC, Negrão JH. Comparison between modal and step-bystep approaches in the optimization of cable-stayed bridges subjected to seismic loads. In: Soc. Mex. Ing. Sismica, ed. 11WCEE, paper/CDROM. Proceedings of the 11th World Conference on Earthquake Engineering, Acapulco, Mexico, Pergamon, UK, 1996.

[8] Kanok-Nukulchai W, Hong G. Nonlinear modelling of cable-stayed bridges. Constructional Steel Research 1993;26:249-66.

[9] Negrão JH, Simões LMC. Cable stretching force optimization in cable-stayed bridges. In: Mróz Z, editor. WCSMO2. Proceedings of the Second World Congress of Structural and Multidisciplinary Optimization, Zakopane, Poland, 1997.

[10] Simões LMC. Information entropy applications in structural optimization. In: Hernandez S, Brebbia CA, editors. OPTI 91. Optimization of structural systems and industrial applications, Cambridge, MA, Elsevier, 1991. p. 15-26

[11] Templeman AB. Entropy-based optimization methods for engineering design. In: Hernández S, editor. Advanced techniques in the optimum design of structures, topics in engineering, vol. 12, Computational Mechanics Publications, UK, 1993. p. 109-39. 